\title{
Fetal metacarpal/metatarsal bone thickness as possible predictor of dystocia in Holstein cows
}

\author{
B. Vincze, ${ }^{*} \dagger$ A. Gáspárdy,† F. L. Kézér, ${ }^{*} \ddagger$ M. Pálffy, ${ }^{*} Z$ s. Bangha,‡ O. Szenci, ${ }^{*}$ and L. Kovács ${ }^{*}{ }^{1}$ \\ *MTA-SZIE Large Animal Clinical Research Group, Üllő-Dóra major H-2225, Hungary \\ †Department of Animal Breeding, Nutrition and Laboratory Animal Science, University of Veterinary Medicine, István utca 2, \\ Budapest $\mathrm{H}-1078$, Hungary \\ ‡Institute of Animal Husbandry, Faculty of Agricultural and Environmental Science, Szent István University, Páter Károly utca 1 , \\ Gödöllö H-2100, Hungary
}

\begin{abstract}
Dystocia and perinatal calf mortality cause significant economic losses in the dairy cattle industry. Despite advanced ultrasound examination procedures, there is no reliable method to estimate the birth weight of calves in order to predict, prepartum, the risk of dystocia. The aim of this study was to predict calf birth weight and dystocia based on transrectal ultrasonographic (TRUS) examinations in late-term Holstein heifers and cows. Therefore, TRUS examination was performed on 128 animals that were between 265 and $282 \mathrm{~d}$ of gestation to measure the bone thickness of the fetal metacarpus (MC) or metatarsus (MT). Fetal TRUS measurements were successful in 104 cases. Excluding twin deliveries, 97 fetal MC/MT bone thicknesses were measured and the mean $( \pm \mathrm{SD}) \mathrm{MC} / \mathrm{MT}$ thickness was $2.54 \pm 0.37$ $\mathrm{cm}$. A novel index, the metacarpal/metatarsal index $[\mathrm{MCTI}=$ maternal body weight $(\mathrm{kg}) /$ fetal MC or MT thickness $(\mathrm{cm})]$, was also calculated to study its association with calving ease. The average MCTI was $257.3 \mathrm{~kg} / \mathrm{cm}$ in the studied population. A lower MCTI was associated with the risk of dystocia with an odds ratio of 2.074 that was not significantly different from 1 (95\% confidence interval: 0.002-11.104). Fetal presentation, fetal age, fetal sex, body condition score of the dam, age of dam, and intercoxal and interischiadic distances were not related to dystocia. A fair phenotypic correlation (0.226) was found between MC/MT thickness and calf birth weight. The genetic correlation between MC/MT thickness and calf birth weight was 0.235 . Our results indicate that late-term measurement of the fetal MC/MT bone thickness by means of TRUS examination augmented with the MCTI may have clinical significance in the prediction of dystocia in Holstein cattle. Because the odds ratio for dystocia based on
\end{abstract}

Received February 27, 2018.

Accepted July 13, 2018.

${ }^{1}$ Corresponding author: Kovacs.Levente@mkk.szie.hu
MCTI determination was not significant, the applied technique should be improved based on further studies on prepartum TRUS examinations combined with dam pelvic measurements.

Key words: dystocia, birth weight, fetal metacarpal/ metatarsal thickness, rectal ultrasonography

\section{INTRODUCTION}

Although an increasing number of studies are investigating the management of perinatal losses caused by difficulties at calving, managing dystocia is still challenging in dairy cattle practice. Therefore, the risk of dystocia should be recognized as early as possible, because early intervention has the potential to prevent stillbirths. The most common causes of dystocia are gestation length and feto-pelvic disproportion (Mee, 2008). The 2 primary determinants of feto-pelvic disproportion are, in order of importance, calf birth weight and maternal pelvic size (Meijering, 1984). Heifers that are bred too young or with a BW that is too low are more likely to experience dystocia. The consequences of dystocia are increased morbidity and mortality rate of newborn calves and subsequent reduced fertility of the dam (Hopper, 2015). Fetal and maternal causes can lead to a difficult birth. Early recognition of the delay in the calving process and intervention can ameliorate the economic impact (Hopper, 2015). In an extensive study, more than half $(51.2 \%)$ of heifers and $29.4 \%$ of cows experienced dystocia (Lombard et al., 2007). In heifers, relative or absolute fetal oversize is a common cause of dystocia (Nakao, 1995), and therefore the estimation of birth weight during fetal life could be a worthwhile effort for practicing veterinarians to avoid perinatal losses.

Although in $5 \%$ of ultrasound examinations the fetus could not be reached at all, out of all fetal body parts, the head remained the most accessible in the case of anterior presentation (Kähn, 1989). In most cases, anterior presentation is observed; therefore, if the head 
is accessible, the metacarpus could be reached with the same success rate. Takahashi et al. (2001) found a strong association between metacarpal widths and calf birth weight measured immediately after birth. Later, fetal metacarpal/metatarsal (MC/MT) width was found to be a useful parameter for estimating birth weight in calves (Takahashi et al., 2005).

Previously, a strong positive genetic association was found between the intrapelvic area and calf birth weight (Benyshek and Little, 1982). The measurement of calf front-hoof circumference and dam pelvic area during stage II of labor has been shown as a diagnostic tool for dystocia (Hiew et al., 2016); however, interventions based on this information are difficult during parturition. We hypothesized that fetal MC/MT thickness would be a reliable diagnostic parameter in predicting calf birth weight or dystocia, and that these parameters would provide valuable information to promote the management of dystocia in dairy cattle.

\section{MATERIALS AND METHODS}

The study was approved by the Department of Epidemiology and Animal Protection of the Directorate of Food Chain Safety and Animal Health at the Central Agricultural Office (Permit Number: PE/EA/19736/2016). All procedures involving animals were approved by the Ethics Committee of the University of Veterinary Medicine.

\section{Animals and Experimental Design}

The experiment was carried out during two 2-d periods in November 2016 on a dairy farm with a herd of 2,300 Holstein cows (Beremend, Hungary; 45 $49^{\prime} 0^{\prime \prime}$ N, $18^{\circ} 28^{\prime} 5.9^{\prime \prime}$ E). From the 240 dried-off, pregnant animals, 58 multiparous cows and 70 heifers were allocated to the study (mean $\pm \mathrm{SD}$; age $=4.2 \pm 1.2 \mathrm{yr}$; parity $=1.7 \pm 0.5$ lactations; day of gestation $=270.4 \pm 7.8$; $\mathrm{BCS}=3.3 \pm 0.2)$ based on the stage of gestation $(10 \mathrm{~d}$ within the expected time of calving).

Animals were kept in a freestall cubicle housing system with sand as the bedding material. A TMR was provided 3 times a day at 0900, 1300, and $1700 \mathrm{~h}$ with a 80:20 forage:concentrate ratio on a DM basis, and water was available ad libitum. The barns were equipped with self-locking headgates along the feeding area, which were used to restrain cows for regular veterinary checkups and the necessary treatments, for a maximum of $2 \mathrm{~h}$. Experimental transrectal ultrasonographic (TRUS) examinations were included in this management schedule and performed during the morning feeding while animals were restrained in the headgates. Thirty-two animals were involved per day and each session required $2 \mathrm{~h}(4 \times 32=128$ total $)$. Each animal was examined only once. Any unnecessary contact with animals throughout the experimental period was avoided.

\section{Ultrasonographical Examination}

Examinations were carried out by the first author. Before the TRUS examination, feces was removed from the rectum. After locating the fetus (if possible), the fetal limbs were fixed by hand to decide whether it was an anterior or posterior presentation. After reentering the rectum with the 5 - to $8-\mathrm{MHz}$ linear-array transducer, the measurement of fetal MC/MT thickness was performed using a portable veterinary ultrasound machine (Mindray M5 Vet, Mindray Medical International Ltd., Shenzhen, China). The thickness of the bone was measured as described by Takahashi et al. (2005). In each case, the thickness was measured 3 times and the mean value was saved into the machine's database (Figure 1). The metacarpal/metatarsal index (MCTI) was counted for every fetus-dam pair as follows:

$$
\mathrm{MCTI}=\frac{\text { Dam BW }(\mathrm{kg})}{\mathrm{MC} \text { or MT bone thickness }(\mathrm{cm})} .
$$

\section{In Vitro Validation of Metacarpal Bone Thickness Measurement}

To test the reliability of fetal parts measurement with TRUS, 3 pairs (left and right) of fetal limbs (cut at the distal point at the carpal joint) were placed into waterfilled rectal gloves and placed into a plastic bucket filled with water. Ten ultrasonographic measurements of the thickness of the MC bone were made on each limb (at the same area of the MC) in the water (Figure 2) with the portable device described above. Then, the bones were cut and the actual bone widths were recorded and compared with the values given by the ultrasonographic measurement. The validation process indicated a 1- to 3-mm difference between the ultrasound measurements and actual bone widths, which is about 3 to $6 \%$ of the total bone width. The regression was shown to be significant $(P=0.04)$ and the correlation coefficient was 0.68 .

\section{Maternal and Calf-Related Data}

Fetal age was calculated from the date of TRUS examination and the date of successful insemination. Fetal sex was determined by the farm veterinarian between d 58 and 65 of gestation, and confirmed immediately after birth during the first clinical examination. The 


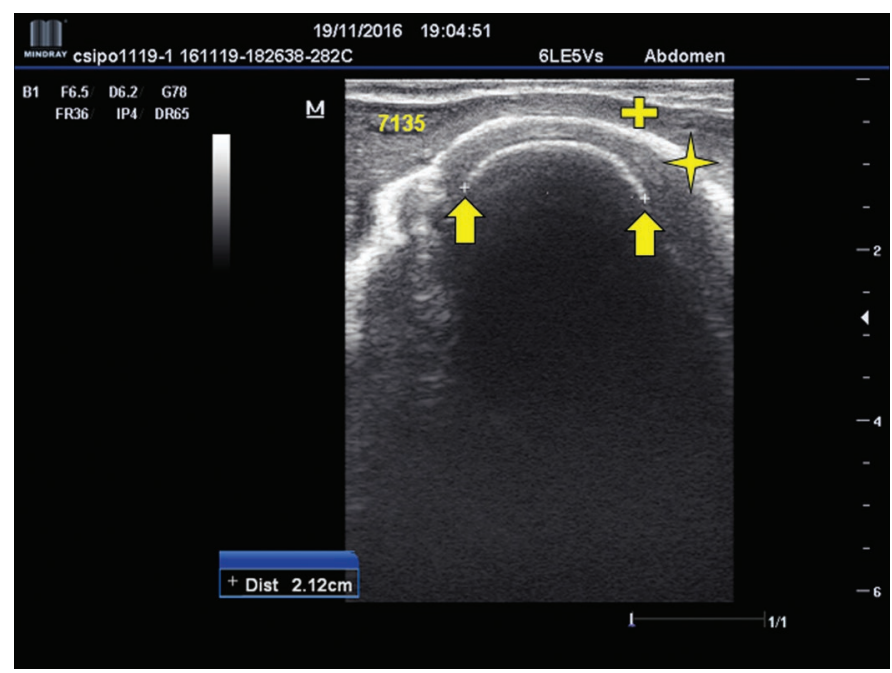

Figure 1. Example of ultrasonographic measurement of fetal metacarpal/metatarsal bone thickness (bone surface to bone surface) obtained with a $5-\mathrm{MHz}$ rectal transducer. Arrows show the 2 endpoints of the metacarpal bone axially and abaxially; the distance between the endpoints is the thickness of the metacarpus (here $2.12 \mathrm{~cm}$ ); the dorsal plane of the sonographic image is the dorsal plane of the fetal limb, the fetal skin (marked with star), and the rectal wall of the dam (marked with plus). Color version available online.

BCS of the dam was scored simultaneously with TRUS examinations using the 5-point scoring system used in the United States (Hady et al., 1994). The BW of the dam was measured after the TRUS examination with a

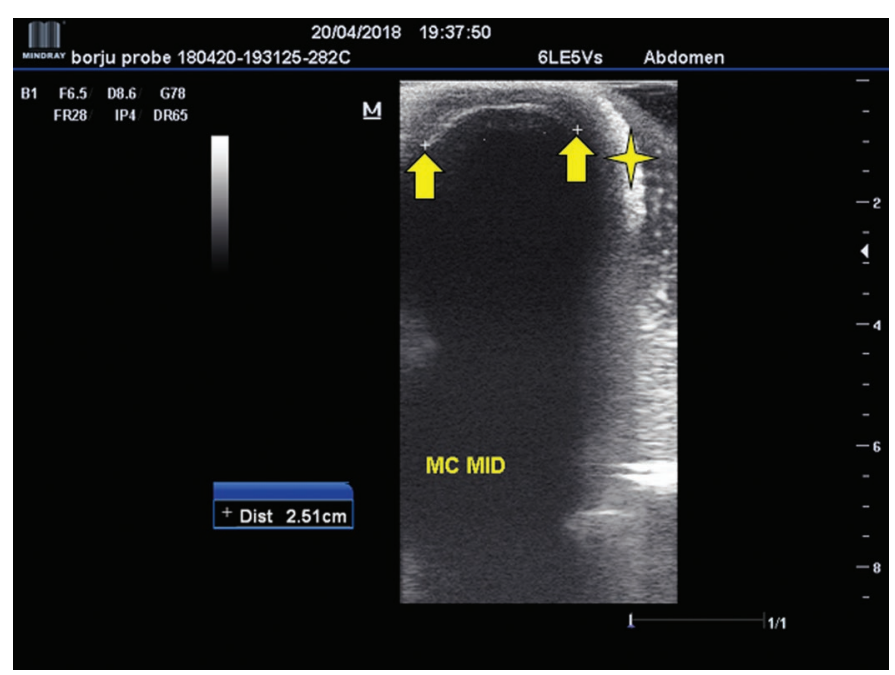

Figure 2. Example of ultrasonographic measurement of fetal metacarpal/metatarsal bone thickness in vitro (bone surface to bone surface) obtained with a $5-\mathrm{MHz}$ rectal transducer. Arrows show the 2 endpoints of the metacarpal bone axially and abaxially; the distance between the endpoints is the thickness of the bone (here $2.51 \mathrm{~cm}$ ); the dorsal plane of the sonographic image is the dorsal plane of the fetal limb and the fetal skin (marked with star). Color version available online. built-in cow weighing scale located in the stall. In addition, the outer intercoxal and interischiadic distances were measured with a simple commercially available tape measure for each animal.

The incidence and degree of dystocia were recorded on a 5-score scale as follows: (1) eutocia (normal calving) was recorded after a combination of no assistance and slight assistance by 1 person (Mee et al., 2011); (2) light dystocia was considered in cases of prolonged spontaneous calvings ( $>2 \mathrm{~h}$ from hooves appearance to delivery) or calvings assisted by 1 person without the use of mechanical traction, with moderate force; (3) mild dystocia required assistance by 2 people without mechanical traction, with considerable force; (4) severe dystocia was recorded after assistance by 3 people with considerable force or assistance with the use of mechanical extraction during delivery (Kovács et al., 2016). During the study period, caesarean section or fetotomy (score 5) were not performed. Eutocic deliveries included calvings with score 1 , and calvings with scores 2, 3 and 4 were dystocic. Excluding twin deliveries, a total of 97 calvings were scored. Calf birth weight was measured immediately after calving.

\section{Statistical Analysis}

Statistica software (version 13; Dell Inc., 2015) was used for data preparation and processing. To evaluate the association between calving ease and the maternal and calf-related parameters, a logistic regression of a generalized linear model was applied. In the logit model, the difficulty of calving (dystocic or eutocic) as dependent variable and fetal presentation, fetal age, fetal sex, BCS of the dam, age of dam, intercoxal and interischiadic distances, and MCTI as independent variables were taken into consideration. Statistical significance of the effects of these traits on calving ease was estimated by a backward elimination, considering which effects were eligible for removal. As a result, the $P$-value of each effect and the maximum likelihood of the model are presented in Table 1. For independent variables that were proven significant, the standard error (SE), 95\% $\mathrm{CI}$, and odds ratio are presented. Other independent variables were presented as mean, standard deviation (SD), and minimum and maximum values.

To evaluate the association between MC/MT bone thickness and calf birth weight, we determined the phenotypic correlation. The association between the corrected values of thicknesses was regarded as the genetic correlation. For both associations, a Spearman rank order correlation $(\rho)$ was additionally used. For this purpose, raw data were adjusted for fetal ages as follows: 270 fetal days for fetal $\mathrm{MC} / \mathrm{MT}$ thickness = actual fetal MC/MT thickness - [(actual fetal age - 
Table 1. $P$-values for maternal and calf-related variables with possible effect on calving ease and the maximum likelihood of the general linear model in the order of removal ${ }^{1}$

\begin{tabular}{llcc}
\hline Independent variable & Order of removal & $P$-value & $\begin{array}{c}\text { Maximum likelihood } \\
\text { (goodness of fit) }\end{array}$ \\
\hline Rump width III & & & 121.30 \\
Rump width I & 1 & 0.90 & 121.32 \\
BCS of the dam & 2 & 0.79 & 121.38 \\
Fetal sex & 3 & 0.60 & 121.66 \\
Age of the dam & 4 & 0.31 & 122.68 \\
Fetal presentation & 5 & 0.38 & 123.48 \\
Fetal age & 6 & 0.31 & 124.54 \\
MCTI $^{4}$ & 7 & 0.20 & 126.26 \\
\hline
\end{tabular}

${ }^{1}$ The order of removal of independent variables was estimated by a backward elimination.

${ }^{2}$ Rump width III = distance between extremities of the tuber ischiadici.

${ }^{3}$ Rump width I = distance between extremities of the tuber coxae.

${ }^{4} \mathrm{MCTI}$ (fetal metacarpal or metatarsal index) = maternal BW $(\mathrm{kg}) /$ fetal metacarpal or metatarsal thickness $(\mathrm{cm})$.

$270) \times 0.0111], \mathrm{r}=0.258, P=0.011$; and 278 fetal days for calf birth weight $=$ actual birth weight $-[($ actual gestation length -278$) \times 0.2241], \mathrm{r}=0.285, P=0.005$. The reason for choosing $270 \mathrm{~d}$ for fetal age was that the TRUS measurement was performed $8 \mathrm{~d}$ before calving, on average. The reason for choosing $278 \mathrm{~d}$ for calf birth weight was the median value of gestation length of the study population. Then, the corrected values of traits were estimated using an individual animal model (Pedigree Viewer, version 6.5, 2015; https://bkinghor .une.edu.au/pedigree.htm). In the selected single-trait models, calf sex was considered a fixed effect, whereas age and BW of the dam at calving were regarded as covariates. Regarding fetal presentation, $89 \mathrm{MC}$ and 8 MT cases were found, which indicates that the incidence of cephalic presentation was $92 \%$. The average MC and MT thickness adjusted for 270 fetal days showed similar values $(2.52$ and $2.58 \mathrm{~cm}$, respectively) without a significant difference $(P=0.691)$; therefore, fetal position was left out of the estimation. The interischiadic distances did not affect the investigated parameters significantly and were also eliminated from the model.

\section{RESULTS}

\section{Outcomes of TRUS Examinations and Calvings}

During the TRUS examinations, a total of 104 fetal limbs could be reached. Among the 104 studied pregnancies, 34 male and 63 female singletons (93.3\%) and 7 twin calvings $(6.7 \%)$ occurred. Two singleton calves were stillborn $(1.9 \%)$. The dystocia rate was $41.2 \%$ from 104 births and $44.3 \%$ from the singleton (experimental) births. Regarding the fetal presentation, $89 \mathrm{MC}$ and $8 \mathrm{MT}$ cases were found, which indicates that the incidence of cephalic presentation was $92 \%$. The coefficient of variation in the 3 consecutive TRUS examinations of fetal MC/MT bone measurements was 0.0411 (97 animals).

\section{Factors Related to Possible Dystocia}

Maternal and calf-related variables having a possible effect on calving ease (labeled 0 for "no/eutocia" ( $\mathrm{n}=$ $57,58.8 \%)$ and 1 for "yes/dystocia" ( $\mathrm{n}=40,41.2 \%)$ are presented for Table 1 in order of removal and decreasing $P$-values. Fetal presentation, fetal age, fetal sex, BCS of the dam, age of dam, and intercoxal and interischiadic distances were not related to dystocia. A lower MCTI was associated with the risk of dystocia with an odds ratio of 2.074 (95\% CI: 0.002 to 11.104 ; percent correct: $60.8 \%$ ), which was not significantly different from 1 . The estimate of the logistic regression for the significant MCTI was (mean \pm SE) $-0.014 \pm$ 0.0064 (95\% CI: -0.0013 to -0.02662$)$. The mean \pm SD (minimum, maximum) MCTI was $257.3 \pm 35.5 \mathrm{~kg} / \mathrm{cm}$ $(163.3,351.9)$.

\section{Association Between MC/MT Thickness and Calf Birth Weight}

Ninety-seven singleton fetal MC/MT bone thicknesses could be measured from the examination of 128 pregnancies $(76 \%)$, and the mean $( \pm \mathrm{SD}) \mathrm{MC} /$ MT thickness was $2.54 \mathrm{~cm}( \pm 0.37 \mathrm{~cm})$. A fair positive genetic correlation $\left(\mathrm{r}_{\mathrm{g}}=0.226, P<0.001\right)$ was found between MC/MT thickness and calf birth weight (Figure 3). Figure 4 shows the genetic correlation $\left(\mathrm{r}_{\mathrm{g}}\right)$ between the corrected values of MC/MT thickness and birth weight. The corrected values fall within a narrower range; however, the medium weak association $\left(\mathrm{r}_{\mathrm{g}}\right.$ 


\section{Metacarpus $/$-tarsus $=1.1813+0.0319^{*}$ birth weight $\mathrm{r}_{\mathrm{p}}=0.4266 ; \quad P<0.001$}

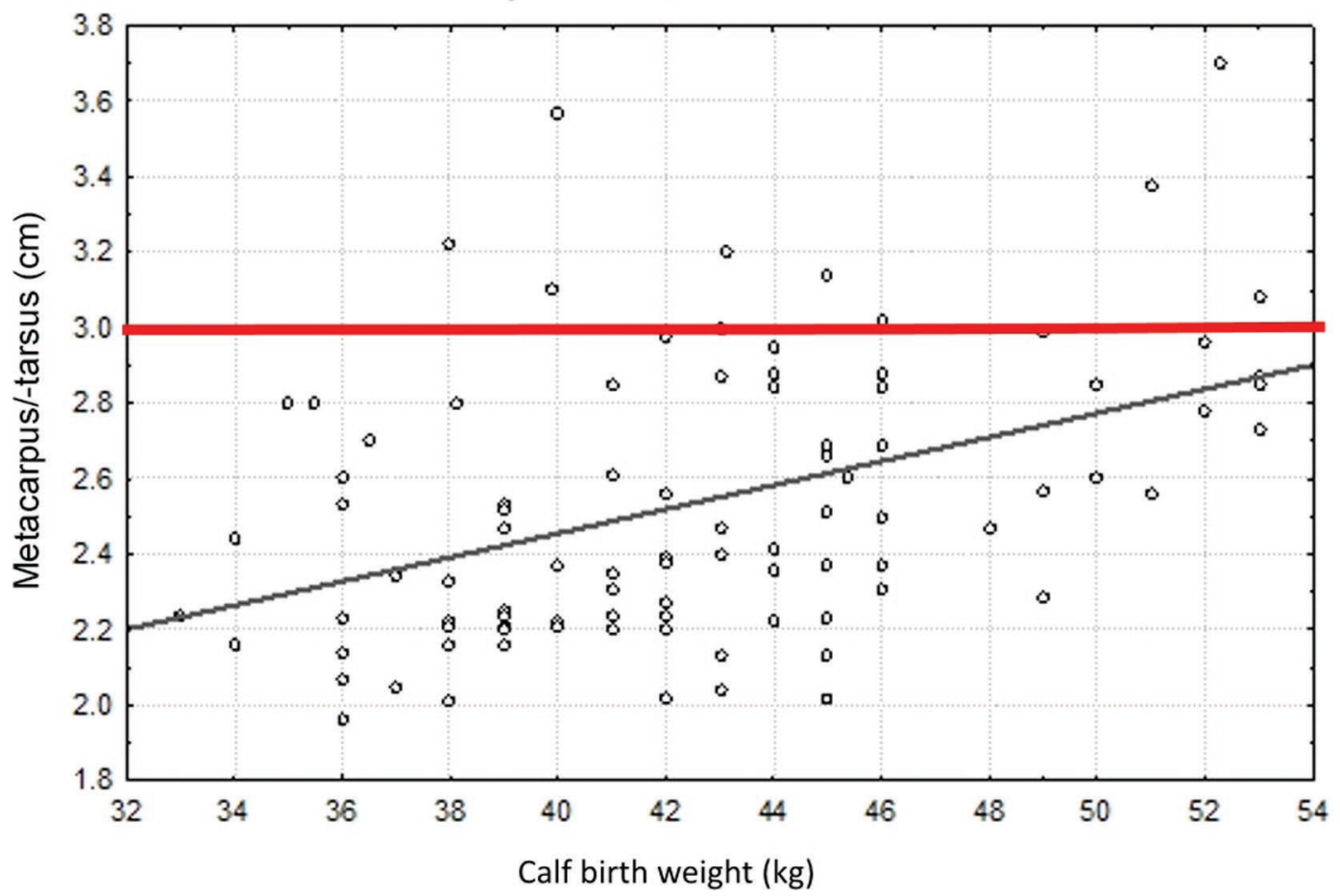

Figure 3. Phenotypic correlation $\left(r_{p}\right)$ between fetal metacarpal/metatarsal thickness and calf birth weight based on raw data. The thick (red) horizontal line at $3.0 \mathrm{~cm}$ indicates the threshold value of metacarpus/metatarsus thickness obtained by Takahashi et al. (2005). Color version available online.

$=0.235)$ was still significant $(P=0.010)$. The Spearman rank correlation was $0.4239(P<0.05)$ between fetal MT/MC bone thickness and calf birth weight, and $0.3559(P<0.05)$ between corrected fetal MT/MC bone thickness and corrected calf birth weight, respectively.

\section{DISCUSSION}

In this study, TRUS examination was performed on 128 heifers and cows to test the applicability of preterm measurement of MC/MT thickness in bovine fetuses. Because late-term pregnant heifers and cows were selected based on their prospective time of calving, we expected that all pregnancies could be examined by transrectal palpation and TRUS, but unforeseen individual differences prevented us from reaching all the fetuses. In 24 of 128 pregnancies examined, the fetal limbs were out of reach from the rectum; therefore, TRUS measurement was not possible. In these cases, the problem was that multiparous cows (19 cases) had a sunken conformation and the fetuses were in a lower position. This is a limiting factor when considering this method as a protocol in routine dairy practice.
According to the significant positive correlation between fetal MC/MT thickness and calf birth weight, $\mathrm{MC} / \mathrm{MT}$ thickness measurement by TRUS should be incorporated in the management of late-term pregnancies, especially in dairy herds where high calf birth weight is a concern. In addition to testing fetal MC/ MT bone thickness as a possible predictor of calf birth weight, our goal was to find a measurable parameter suitable for predicting dystocic calving events in dairy cattle.

Based on our results, a lower MCTI calculated between 265 and $282 \mathrm{~d}$ of gestation seems to be associated with a higher risk of dystocia. The odds ratio obtained was about 2.0, and we concluded that dam-fetus pairs characterized with MCTI $<257 \mathrm{~kg} / \mathrm{cm}$ tended to be twice as likely to experience a dystocic calving.

The nonsignificant odds ratio of approximately 2 suggest that the applied technique should be refined based on results of further large-scale studies evaluating the best predictor of either extra- or intra-pelvic dam pelvic size. Because the primary cause of dystocia in cattle is fetal-dam disparity, several attempts have been made to predict calving ease based upon 
pelvimetry (Doornbos et al., 1984; Murray et al., 2002; Hiew et al., 2016). Intra-pelvic measurements (such as intra-pelvic area) can be accurate to examine the size of the birth canal (Murray et al., 2002; Hiew et al., 2016); however, the use of a pelvimeter (e.g., Rice pelvimeter) is considered an invasive procedure that requires epidural anesthesia for correct values without straining or holding an arched back of the cow (Murray et al., 2002). In an early report, pelvic measurements were found reasonable and $80 \%$ reliable, and commercially available instruments were found to be easy and affordable to use (Daly and Riese, 1992). Although Murray et al. (2002) demonstrated that, in Belgian Blue cows, external pelvic measures correlated with internal pelvic width and length, the correlation was not significant for all examined groups in that study. Previously, measurement of the yearling female was performed, but unfortunately, heifers with larger pelvic openings tend to be larger in frame score, having calves with heavier birth weight; therefore, selecting for larger openings does not lead to the reduction of dystocia rate (Hopper, 2015). Pelvic area and calf birth weight ratios have been calculated for different replacement heifer weights to lower the occurrence of dystocia cases on beef cattle farms (Johnson et al., 1988). Studies showed that early intervention yields more live healthy calves and more live healthy heifers with improved reproductive rate compared with later intervention (Doornbos et al., 1984).

In conclusion, the MCTI seems to be a promising approach in predicting dystocia at the individual level. Farms experiencing higher rates of heifer dystocia could use this technique in establishing their own, farm-tailored heifer development plan. Nevertheless, MCTI as a dystocia prediction method is limited by the fact that the test of the present study might have too much variability as dystocia has a multifactorial nature and we cannot predict all dystocia cases. Dystocia rates are highly influenced by calving management and by the case definition of dystocia. Furthermore, the critical MCTI value reported in the present study is only correct for the population studied and needs to be re-evaluated in the target population. Therefore, before MCTI can be recommended for practical use, experiments should test the efficiency of MCTI in dairy farms with different parturition management characteristics in predicting dystocia. We suggest using the same dystocia scoring system in further assessment of MCTI that should be supplemented with pelvic measures. Based on the results of these investigations, the efficiency of late-term interventions (i.e., calving inductions) in decreasing the prevalence of dystocia could be

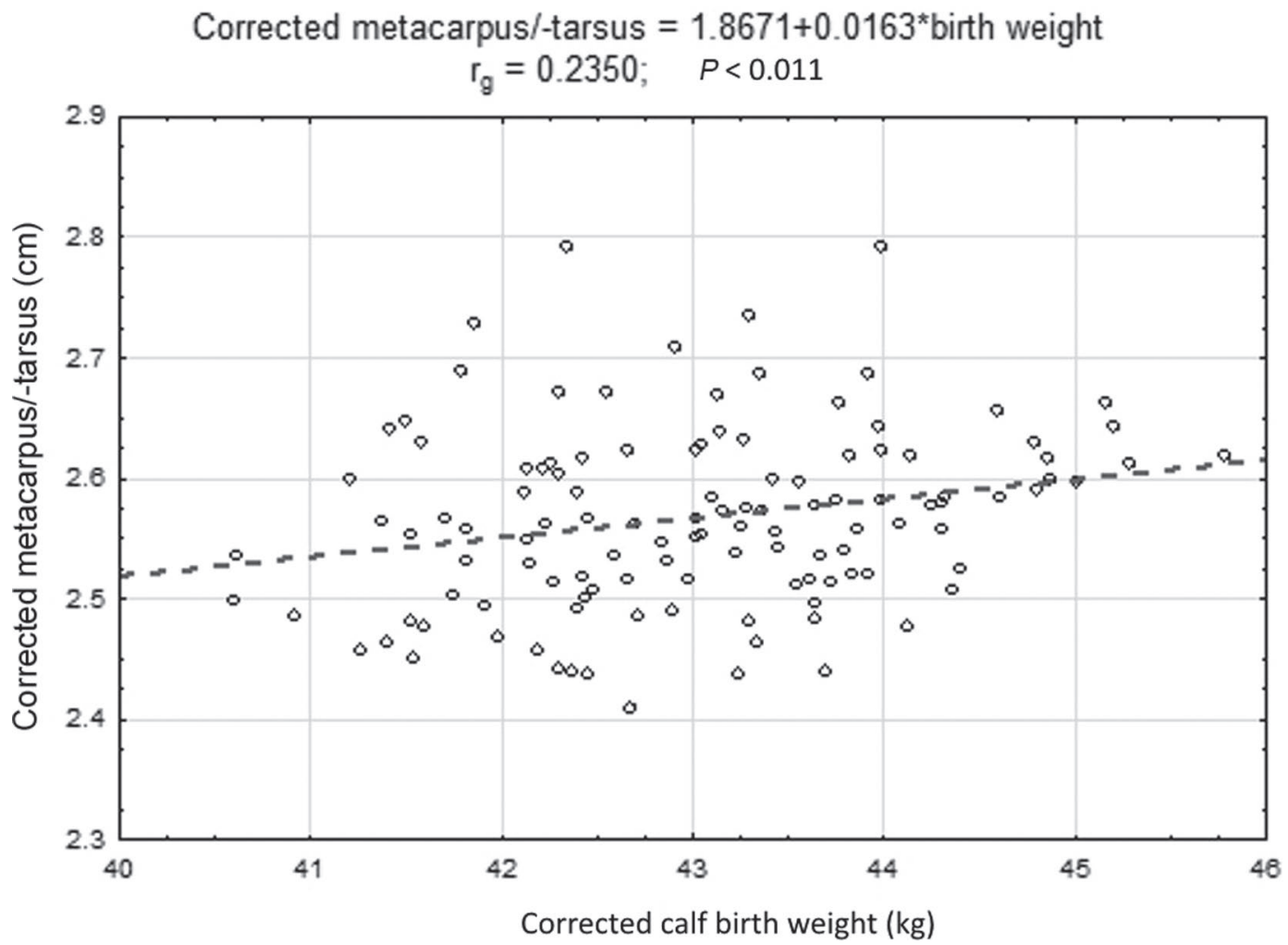

Figure 4. Genetic correlation $\left(\mathrm{r}_{\mathrm{g}}\right)$ between fetal metacarpal/metatarsal thickness and calf birth weight based on corrected values. 
tested in dairy herds where calf-dam mismatch or large calf birth weight are of concern.

\section{ACKNOWLEDGMENTS}

Levente Kovács was supported by the János Bolyai Research Scholarship of the Hungarian Academy of Sciences, Hungary (BO/00040/16/4); OTKA Postdoctoral Scholarship of the National Research, Development and Innovation Office, Hungary (NKFIH-6493-1/2016); Research Center of Excellence project of the National Research, Development and Innovation Office, Hungary (1476-4/2016/FEKUT); and New National Excellence Program of the Ministry of Human Capacities (ÚNKP17-4-I/SZIE-7).

\section{REFERENCES}

Benyshek, L. L., and D. E. Little. 1982. Estimates of genetic and phenotypic parameters associated with pelvic area in Simmental cattle. J. Anim. Sci. 54:258-263.

Daly, R. F., and R. L. Riese. 1992. Pelvic measurements: Applications in beef cattle practice today. Iowa State Univ. Vet. 54:44-51.

Dell Inc. 2015. Dell Statistica data analysis software system. Version 13. Dell Software/Tibco Software Inc., Palo Alto, CA.

Doornbos, D. E., R. Bellows, P. Burfening, and B. Knapp. 1984. Effect of dam age, prepartum nutrition and duration of labor on productivity and postpartum reproduction in beef females. J. Anim. Sci. $59: 1-10$.

Hady, P. J., J. J. Domecq, and J. B. Kaneene. 1994. Frequency and precision of body condition scoring in dairy cattle. J. Dairy Sci. $77: 1543-1547$.

Hiew, M. W. H., A. A. Megahed, J. R. Townsend, W. L. Singleton, and P. D. Constable. 2016. Clinical utility of calf front hoof circumfer- ence and maternal intrapelvic area in predicting dystocia in 103 late gestation Holstein-Friesian heifers and cows. Theriogenology $85: 384-395$.

Hopper, R. M. 2015. Management to prevent dystocia. Pages 404-416 in Bovine Reproduction. R. M. Hopper, ed. Wiley \& Sons, Ames, IA.

Johnson, S. K., G. H. Deutscher, and A. Parkhurst. 1988. Relationships of pelvic structure, body measurement pelvic area and calving difficulty. J. Anim. Sci. 66:1081-1088.

Kähn, W. 1989. Sonographic fetometry in the bovine. Theriogenology 31:1105-1121.

Kovács, L., F. L. Kézér, and O. Szenci. 2016. Effect of calving process on the outcomes of delivery and postpartum health of dairy cows with unassisted and assisted calvings. J. Dairy Sci. 99:7568-7573.

Lombard, J. E., F. B. Garry, S. E. Tomlinson, and L. P. Garber. 2007. Impacts of dystocia on health and survival of dairy calves. J. Dairy Sci. 90:1751-1760.

Mee, J. F. 2008. Prevalence and risk factors for dystocia in dairy cattle: A review. Vet. J. 176:93-101.

Mee, J. F., D. P. Berry, and A. R. Cromie. 2011. Risk factors for calving assistance and dystocia in pasture-based Holstein-Friesian heifers and cows in Ireland. Vet. J. 187:189-194.

Meijering, A. 1984. Dystocia and stillbirth in cattle-A review of causes, relations and implications. Livest. Prod. Sci. 11:143-177.

Murray, R. D., T. A. Cartwright, D. Y. Downham, M. A. Murray, and A. de Kruif. 2002. Comparison of external and internal pelvic measurements of Belgian blue cattle from sample herds in Belgium and the United Kingdom. Reprod. Domest. Anim. 37:1-7.

Nakao, T. 1995. Dystocia. Pages 369-387 in Textbook of Theriogenology. J. Mori, H. Kanagawa, and K. Hamana, ed. Buneido Publishing Co. Ltd., Tokyo, Japan.

Takahashi, M., T. Goto, H. Tsuchiya, A. Ueki, and K. Kawahata. 2005. Ultrasonographic monitoring of nuclear transferred fetal weight during the final stage of gestation of Holstein cow. J. Vet. Med. Sci. 67:807-811.

Takahashi, M., A. Ueki, K. Kawahata, and T. Goto. 2001. Relationships between the width of metacarpus or metatarsus and the birth weight in Holstein calves. J. Reprod. Dev. 47:105-108. 